\title{
Joint Beamforming and Power Allocation Cognitive Radio Networks under Imperfect CSI
}

\author{
Jie Chen ${ }^{1,2}$, Wen $\mathrm{Xi}^{2}$ and Xiaolong Sun $^{2}$ \\ ${ }^{1}$ School of Electro-Mechanical Engineering, Xidian University, Xian 710071, \\ China \\ ${ }^{2}$ Chang jiang Computer Group Corporation, Shanghai 200001, China

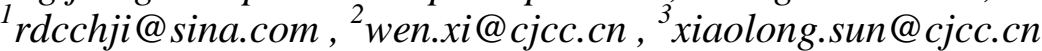

\begin{abstract}
Traditional beamforming and power control algo-rithms in cognitive radio (CR) are based on the assumption of perfect channel state information (CSI) however; this may lead to performance degradation in realistic systems. In this paper, the problem of joint beamforming and power control is investigated in underlay CR networks with imperfect CSI. Our objective is to maximize the sum utility of secondary users (SUs) under the primary users (PUs) interference power constraints and the transmission power constraint of SUs. First, the joint beamforming and power control problem is formulated under game theory framework, where the SUs compete with each other over the beamforming vectors and transmission power level made available by the PUs. Moreover, the channel uncertainty is described using ellipsoid sets and the interference power constraints can be converted into robust interference power constraints. Besides, Nash equilibrium (NE) is considered as the solution of this game. Finally, simulation results show that the proposed scheme can converge to a locally optimal pair of beamforming vector and transmission power level in the presence of channel uncertainty.
\end{abstract}

Keywords: Cognitive radio, beamforming, power allocation, imperfect channel state information, Nash equilibrium.

\section{Introduction}

COGNITIVE radio (CR), as a promising technology to enhancing the utilization efficiency of the scarce radio spectrum, has attracted tremendous interests recently. A key feature of the CR network is to allow a secondary user (SU) to simultaneously share a licensed spectrum as long as the secondary transmission does not interfere with the primary link. As a result, the challenge of the CR network is to protect the primary users (PUs) from harmful interference induced by the SUs as well as to meet the quality of service (QoS) demands of SUs [1].

Cognitive beamforming and power control, as an effective interference suppression technology, has been widely used in CR from different aspects [2-4]. All these work are based on the assumption of perfect channel knowlege. However, in practical systems, perfect CSI is difficult to obtain due to the loose cooperation between PUs and SUs, as well as many other factors such as inaccurate channel estimation, limited feedback or lack of channel reciprocity. The worst-case approach has been used to design robust power for SUs in a multiple-input single-output (MISO) CR system [5, 6]. In [5], the software assisted method and a geometric method were considered for single SU and single PU to find suboptimal solution for the certainty and uncertainty models. A bounded region for channel matrices and channel covariance matrices was assumed to be known in [6]. The authors used a type of ellipsoid uncertainty problem to express the bound channel uncertainty. 
For more SUs or PUs, [7] made some approximations for the uncertainty channel model between SUs and PUs. In [8], the worst-case of uncertainty was considered and the initially non-convex uncertainty problems are transformed into a second order cone programming (SOCP) or other convex problems, which can be solved by software. In [9], a linear precoder design scheme based on the minimum mean square error (MMSE) criteria for a CR multi-input multi-output (MIMO) system was presented. The proposed scheme, with the consideration of both cooperative feedback from PUs and local feedback from SUs to the secondary base station (SBS), is robust to the channel uncertainties. Robust distributed power allocation algorithm for underlay CR networks was proposed in [10], which maximizes the sum utility of SUs when channel gains from SUs to primary base station (PBS) and interference introduced by PUs to the SBS are uncertain. In [11], the authors studied the problem of joint beamforming and power allocation in a cognitive MIMO system using game theory, where the imperfect CSI was taken into account by the robust interference constraint and the optimization problem in the formulated robust game is converted into a SOCP problem.

Inspired by the aforementioned work, in this paper, we consider the problem of joint beamforming and power control for underlay CR via game theory under imperfect CSI, where multiple SUs coexisting with multiple PUs. The objective is to maximize the sum utility of SUs under the interference power constraints at PUs, the total transmission power constraint of SUs, and SINR constraint of each SU. The joint problem is formulated as non-cooperative game, and then an ellipsoid model is adopted to describe the CSI uncertainty. By taking some approximation, the problem is reformulated as a SOCP problem. Simulation results show that the effectiveness of our proposed scheme.

The reminder of this paper is organized as follows. In section II, the system mode of $\mathrm{CR}$ is introduced and the optimization problem under imperfect CSI is formulated. In section III, the problem of joint beamforming and power control is formulated as noncooperative game, and then the problem is transformed into a SOCP problem. In Section IV, numerical results are shown for our scheme. Finally, Section V draws some concluding remarks.

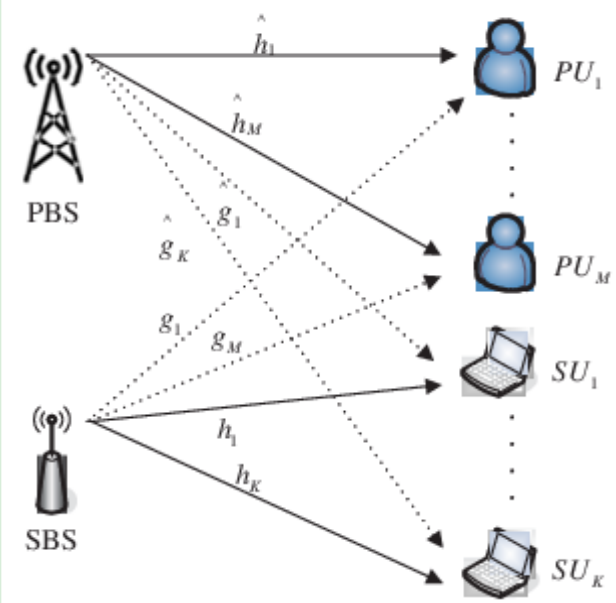

Figure 1. Cognitive Radio System Model. Solid line Denotes Transmission Channel and Dotted line Denotes Interference Channel

\section{System Model and Problem Formulation}

As illustrated in Fig. 1, we consider a cognitive network where a primary network consisting of a PBS and M Pus coexists with a secondary network with a SBS and K SUs. In the secondary network, SUs operate in the frequency band allocated to the PUs, thus the channels between the base stations and users are inherently interference channels. We 
assume that SBS has $N_{t}$ antennas, PBS has a single antenna, and each SU and PU are equipped with only one antenna. For the sake of simplicity, we assume a block fading model for all channels. Let $h_{k} \in C^{1 \times N_{t}}, k \in[1, K]$, and $g_{m} \in C^{1 \times N_{t}},, m \in[1, \mathbf{M}]$ denote the channel gains from the SBS to $S U_{k}$ and to $P U_{m}$, respectively. Likewise, channel gains between PBS and $P U_{m}$ and between PBS and $S U_{k}$ are denoted by $\hat{h}_{m}$ and $\hat{g}_{k}$, respectively. As such, the received signal at $S U_{k}$ is represented as

$$
y_{k}=\sqrt{p_{k}} h_{k} f_{k} s_{k}+h_{k} \sum_{i=1, i \neq k}^{K} \sqrt{p_{i}} f_{i} s_{i}+\hat{g}_{k} \sum_{m=1}^{M} \sqrt{p_{m}} s_{m}+n_{k}
$$

where $s_{k}$ and $s_{\mathrm{m}}$ are the transmitted signals from SBS to $S U_{k}$ and that from PBS to $P U_{m}$, respectively. Likewise, $p_{k}$ and $p_{m}$ account for the transmission power of $S U_{k}$ and $P U_{m} . f_{k}$ denotes the beamforming vectors of $S U_{k}$ and PU. And $n_{k}$ is an additive white Gaussian noise with zero mean and variance $\sigma_{k}^{2}$. The signal-to-interference-plus-noiseratio (SINR) of the k-th SU is calculated by

$$
\operatorname{SINR}_{k}=\frac{\left|h_{k} f_{k}\right|^{2} p_{k}}{\sum_{i=1, i \neq k}^{K}\left|h_{k} f_{\mathrm{i}}\right|^{2} p_{i}+\sum_{m=1}^{M}\left|\hat{g}_{k}\right|^{2} p_{m}+\sigma_{k}^{2}}
$$

In the cognitive network, to guarantee the QoS requirements of $S U_{S}$, the received SINR of each SU should be greater than a threshold $\gamma_{k}$ [6], i.e.,

$$
\operatorname{SINR}_{k}=\gamma_{k}
$$

On the other hand, to enable the reliability of communi-cations of $S U_{S}$, interference power introduced by SUs should not exceed a predefined threshold $p_{m, t h}$ at $P U_{m}$. Therefore, the total received power at a specified $P U_{m}$ must meet [4]

$$
\sum_{k=1}^{K}\left|g_{m} f_{k}\right|^{2} p_{k} \leq p_{m, t h}
$$

The achievable rate at $S U_{k}$ is given by

$$
\begin{aligned}
\mathbf{R}_{k} & =\log \left(1+\operatorname{SINR}_{k}\right) \\
& =\log \left(1+\frac{\left|h_{k} f_{k}\right|^{2} p_{k}}{\sum_{i=1, i \neq k}^{K}\left|h_{k} f_{\mathrm{i}}\right|^{2} p_{i}+\sum_{m=1}^{M}\left|\hat{g}_{k}\right|^{2} p_{m}+\sigma_{k}^{2}}\right.
\end{aligned}
$$

where $\sum_{m=1}^{\mathrm{M}}\left|\hat{g}_{k}\right|^{2} p_{m}$ is the interference introduced by PUs.

To improve the system performance of the secondary net-work, we employ a joint beamforming and power allocation strategy to maximize the sum rate of SUs under the interference constraints at PUs, the total transmission power constraint of SUs, and the SINR constraint of each SU. The optimization problem can be formulated as follows 


$$
\begin{aligned}
& \max _{f_{k}, p_{k}} \sum_{k=1}^{K} R_{k} \\
& \text { S.t. } \sum_{k=1}^{K}\left|g_{m} f_{k}\right|^{2} p_{k} \leq p_{m, t h}, \\
& \sum_{k=1}^{K} p_{k} \leq P_{T}, \\
& \operatorname{SINR}_{k} \geq \gamma_{k}
\end{aligned}
$$

However, in practice, the CSI between the SBS and the PU is often imperfect due to lack of cooperation. Under ellipsoid approximation [11], the true channel coefficient gm can be written as

$$
g_{m}=\tilde{g}_{m}+\Delta m
$$

where $\tilde{g}_{m}$ is the estimated channel vector at the SBS and $\Delta m$ is a norm-bounded uncertain vector, namely,

$$
|\Delta m|^{2} \leq c_{m} \varepsilon_{m}^{2}
$$

Here, $c_{m}$ and $\varepsilon_{m}^{2}$ are uncertainty parameter and estimation error, respectively.

By considering the channel uncertainty between PU and SBS, the optimization problem (6) can be rewritten as

$$
\begin{aligned}
& \max _{f_{k}, p_{k}} \sum_{k=1}^{K} R_{k} \\
& \text { s.t. } \sum_{k=1}^{K}\left|\left(\tilde{g}_{m}+\Delta m\right) f_{k}\right|^{2} p_{k} \leq p_{m, t h}, \\
& \sum_{k=1}^{K} p_{k} \leq P_{T}, \\
& \operatorname{SINR} R_{k} \geq \gamma_{k}, \\
& |\Delta m|^{2} \leq c_{m} \varepsilon_{m}^{2} .
\end{aligned}
$$

\section{Game under Imperfect CSI}

Under the assumption of imperfect CSI between the SBS and PUs, in order to enable the SUs to share the spectrum with PUs, we should find appropriate power level and beamforming vectors to distribute them among the SUs so that the sum utility of SUs is maximized and the interference created to PUs is as low as possible. In this section, the problem of joint beamforming and power allocation under imperfect CSI will be described from the perspective of game theory.

\subsection{Game-theoretic Formulation}

Game theory is an effective tool to deal with the strategy choice and balancing among individuals who are of conflict interests. The players in a game with conflict interests will selfishly choose their own strategies to maximize their utilityfunctions. In CR network, SUs share the spectrum bands of PUs in a competitive way, which will inevitably interfere with each other [12]. Therefore, the problem of joint beam- forming and power 
allocation is viewed as a non-cooperative game. Based on the system model described earlier, the non- cooperative game can be formulated as

$$
G=\left[N,\left\{f_{i}, p_{i}\right\}_{i \in N},\left\{u_{i}(\cdot)\right\}_{i \in N}\right.
$$

and it has the following three major components.

1) Players: In this paper, players are SUs. A finite set of sensor nodes is denoted as $N=\{1,2, \cdots, K\}$.

2) Strategic Space: The strategic space is defined by the beamforming strategies $f_{i}$ and power strategies $p_{i}$ of SUs. The joint strategy spaces are defined as $\theta=f_{1} \times f_{2} \times \cdots \times f_{K}$ and $P=p_{1} \times p_{2} \times \cdots \times p_{K}$.

3) Utility Function: In this paper, $u_{i}$ is the corresponding utility function of $\mathrm{SU}_{i}$.

A utility function of a player will assign numbers for every possible outcome in the game. In general, a higher number normally implies a more preferred outcome. The utility function can be designed based on the achievable rate, i.e.,

$$
u_{\mathrm{k}}=\log _{2}\left(1+\operatorname{SINR}_{k}\right)
$$

\subsection{Second Order Cone Programming Solution}

In this subsection, the optimization problem (9) is solved via a SOCP solution. As is known to all, the zero-forcing (ZF) scheme is a simple and efficient beamforming method which maximizes the sum utility by choosing appropriate $f_{k}$ [14]. Here, we adopt the ZF beamforming that transforms the broadcast channel into multi-parallel independent and orthog-onal sub-channels. The beamforming vectors are selected to satisfy $h_{k} f_{i}=0, i \neq k$. Suppose that $F=\left[f_{1}, f_{2}, \cdots, f_{K}\right]$ denotes the beamforming matrix, one easy way to choose the beamforming matrix $\mathrm{F}$ that gives the zero interference is as follows [15]

$$
F=H^{H}\left(H H^{H}\right)^{-1}
$$

where $H=\left[h_{1}, \cdots, h_{K}\right]$ denotes the channel matrix and $(.)^{H}$ denotes the conjugate transpose operation. Here, $\mathrm{F}$ is the Moore-Penrose pseudoinverse of the channel matrix $\mathrm{H}$. Therefore, the SINR in (2) becomes

$$
\operatorname{SINR}_{k}=\frac{\left|h_{k} f_{k}\right|^{2} p_{k}}{\sum_{m=1}^{M}\left|\hat{g}_{k}\right|^{2} p_{m}+\sigma_{k}^{2}}
$$

Based on (12) and (13), we can perform the power allocation for SUs and rewrite the optimization problem in (9) as

$$
\begin{aligned}
& \max _{p_{k}, f_{k}} \sum_{k=1}^{K} \log \left(1+k_{k} p_{k}\right) \\
& \text { s.t }\left|\left(\tilde{g}_{m}+\Delta m\right) f_{k}\right|^{2} p_{k} \leq p_{m, t h}, \\
& \sum_{k=1}^{K} p_{k} \leq P_{T},
\end{aligned}
$$




$$
\begin{aligned}
& R_{k} p_{k} \geq \gamma_{k}, \\
& \|\Delta m\|^{2} \leq c_{m} \varepsilon_{m}^{2} \text {. } \\
& \text { Where } R_{k}=\frac{\left|h_{k} f_{k}\right|^{2}}{\sum_{m=1}^{M}\left|\hat{g}_{k}\right|^{2} p_{m}+\sigma_{k}^{2}}
\end{aligned}
$$

Obviously, the optimization problem is non-convex. After we make some approximations, the objective function is equivalent to maximizing $R_{k} p_{k}$ [16]. By defining $t_{k}=\sqrt{p_{k}} f_{k}$, the objective function can be rewritten as $\sum_{k=1}^{K}\left|h_{k} t_{k}\right|^{2}$. Similarly, the interference power of $P U_{m}$ can be expressed as $\sum_{k=1}^{K}\left|\left(\tilde{g}_{m}+\Delta m\right) t_{k}\right|^{2}$. Thus, the problem (14) can be transformed into

$$
\begin{aligned}
& \max _{p_{k}, f_{k}} \sum_{k=1}^{K}\left|h_{k} t_{k}\right|^{2} \\
& \text { s.t. } \sum_{k=1}^{K}\left|\left(\tilde{g}_{m}+\tilde{\Delta} m\right) t_{k}\right| \leq \sqrt{p_{m, t h}} \\
& \sum_{k=1}^{K} p_{k} \leq P_{T}, \\
& \frac{\left|h_{k} t_{k}\right|^{2}}{\sum_{m=1}^{M}\left|\hat{g}_{k}\right|^{2} p_{m}+\sigma_{k}^{2}} \geq \gamma_{k}, \\
& \|\Delta m\|^{2} \leq c_{m} \varepsilon_{m}^{2} .
\end{aligned}
$$

Now we show that the above problem can be reformulated as a second order cone program (SOCP) problem. Defining $\tilde{\Delta} m=\frac{1}{\sqrt{c_{m}}} \Delta m$, the interference constraint in (15) is equivalent to

$$
\sum_{k=1}^{K} \max _{\|\hat{\Delta} m\| \leq \varepsilon_{m}}\left|\left(\tilde{g}_{m}+\tilde{\Delta} m\right) t_{k}\right| \leq \sqrt{p_{m, t h}}
$$

Using the triangle inequality and the Cauchy-Schwarz inequality with $\|\tilde{\Delta} m\| \leq \varepsilon_{m}$, it follows that

where the equality is achieved when

$$
\begin{aligned}
\sum_{k=1}^{K}\left|\tilde{g}_{m} t_{k}+\tilde{\Delta} m \sqrt{c_{m}} t_{k}\right| & \leq \sum_{k=1}^{K}\left(\left|\tilde{g}_{m} W_{k}\right|+\left|\tilde{\Delta} m \sqrt{c_{m}} t_{k}\right|\right) \\
& \leq \sum_{k=1}^{K}\left|\tilde{g}_{m} t_{k}\right|+\left|\tilde{\Delta} m \sqrt{c_{m}} t_{k}\right| \\
& \leq \sum_{k=1}^{K}\left|\tilde{g}_{m} t_{k}\right|+\varepsilon_{m}|| \sqrt{c_{m}} t_{k}||
\end{aligned}
$$

$$
\tilde{\Delta} m=\varepsilon_{m} e^{j \phi_{m}} \frac{\sqrt{c_{m}} t_{k}}{\left\|\sqrt{c_{m}} t_{k}\right\|}
$$

where $\phi_{m}=\angle\left(\tilde{g}_{m} t_{k}\right)$. This indicates 


$$
\sum_{k=1}^{K} \max _{\|\underline{\Delta} m\| \leq \varepsilon_{m}}\left|\left(\tilde{g}_{m}+\tilde{\Delta}_{m}\right) t_{k}\right|=\sum_{k=1}^{K}\left|\tilde{g}_{m} t_{k}\right|+\varepsilon_{m}\left\|\sqrt{c_{m}} t_{k}\right\|
$$

So (16) is equivalent to

$$
\sum_{k=1}^{K}\left|\tilde{g}_{m} t_{k}\right|+\varepsilon_{m} \| \sqrt{c_{m}} t_{k} \mid \leq \sqrt{p_{m, t h}}
$$

Note that the arbitrary phase rotation of $t_{k}$ does not change the value of the objective function or the constraints. Therefore, we can assume that $t_{k}, h_{k}, g_{m}$ have the same phase, i.e.,

$$
\operatorname{Re}\left\{h_{k} t_{k}\right\} \geq 0, \operatorname{Im}\left\{h_{k} t_{k}\right\}=0, \operatorname{Im}\left\{\widetilde{g}_{m} t_{k}\right\}=0
$$

By combining (15), (20), and (21), the optimization problem can be converted into a SOCP problem, as

$$
\begin{aligned}
& \max _{p_{k}, f_{k}} \sum_{k=1}^{K} h_{k} t_{k} \\
& \text { s.t. } \operatorname{Im}\left\{\tilde{g}_{m} t_{k}\right\}=0, \\
& \sum_{k=1}^{K}\left(\left|\tilde{g}_{m} t_{k}\right|+\sqrt{c_{m}} \| t_{k} \mid\right) \leq \sqrt{p_{m, t h}}, \\
& \sum_{k=1}^{K}\left\|t_{k}\right\| \leq \sqrt{P_{T}}, \\
& \frac{\left|h_{k} t_{k}\right|^{2}}{\sum_{m=1}^{M}\left|\hat{g}_{k}\right|^{2} p_{m}+\sigma_{k}^{2}} \geq \gamma_{k} .
\end{aligned}
$$

Similar to [10], we can prove that the joint beamforming and power allocation problem (22) is a convex optimization problem with respect to $t_{k}$ [13]. However, this optimization problem is very difficult to solve, we will resort to numerical simulations to illustrate the convergence property of the sum rate of SUs.

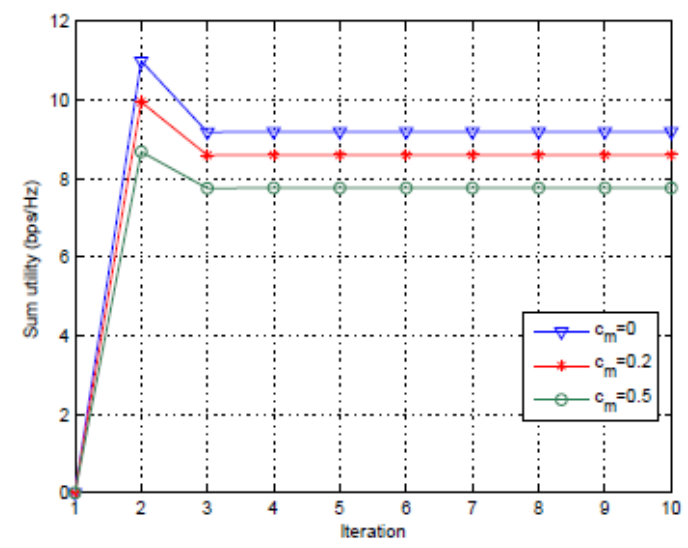

Figure 2. Sum utility of SUs for different $\varepsilon_{m}$

\subsection{Analysis of NE}

Due to $\operatorname{Im}\left\{h_{k} t_{k}\right\}=0,\left[h_{1}, \cdots, h_{k}\right]$ is not of full column rank [17]. However, the best response of each SU is a dominant strategy (Generally, if the feasible strategy for a player 
is a dominant strategy, regardless of the strategies of the other players, its strategy will maximize the payoff function of the players). This implies that the above game has a unique NE.

\section{Numerical Results}

In this section, simulation results are presented to evaluate the performance of the proposed scheme under Rayleigh flat fading channels. We assumed that the SBS has imperfect CSI from PU, and the sum utility achieved by a SOCP approximation algorithm is evaluated [11]. we consider the CR network with four SUs and two PUs, transmitter antennas $N_{t}=4$. In the following results, we choose PUs transmit power as $p_{1}=p_{2}=0.1 \mathrm{~W}$, the $\mathrm{SU}$ maximum transmit power as $P_{\max }=20 \mathrm{~W}$, the interference threshold total transmission power of SBS as $P_{T}=20 \mathrm{~W}$, the interference threshold as $p_{1, t h}=p_{2, t h}=10 \mathrm{~W}$, the k-th SU minimum SINR constraint as $\gamma_{k}=5 d B$, the noise power as $\sigma^{2}=3 \times 10^{-3} \mathrm{~W}$, respectively.

In Fig. 2, the estimation error is set to $\varepsilon_{m}=\left\|\tilde{g}_{m}\right\|^{2}$. Fig. 2 depicts the sum utility of SUs with respect to iteration number. It can be observed that the sum utility of SUs decrease due to uncertainty parameter $\mathrm{cm}$ increases. And the convergence means that the game reaches an equilibrium state.

Fig. 3 shows the sum utility of SUs as a function of transmission power for a setup with $c_{m}=[0,0.005,0.2,0.5]$. When $c_{m}=0$, the CSI is perfect. Intuitively, there will be an optimal value of $p_{k}$ with the increase of transmission power constraint of SUs under imperfect CSI. We can observe that, compared to the perfect CSI, the sum utility under imperfect CSI is lower. What's more, as the value of the uncertainty parameter increasing, the sum utility of SUs becomes less, which is mainly caused by the CSI uncertainty.

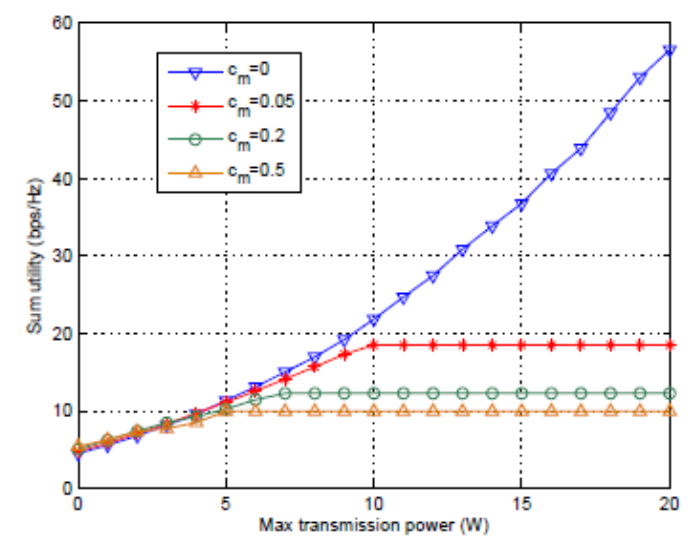

Figure 3. Sum Utility of SUs under Perfect CSI and Imperfect CSI

\section{Conclusion}

In this paper, the problem of joint beamforming and power control in underlay CR with multiple PUs and multiple SUs was studied. Imperfect CSI between the SBS and PUs was considered. The problem was formulated as non-cooperative game, and then an ellipsoid model was adopted to describe the CSI uncertainty. After making some approximations, the problem was reformulated as a SOCP problem. Simulation results shown that the proposed scheme converges to an equilibrium state. And the sum utilities of SUs were also presented to illustrate the performance of the secondary network under perfect and imperfect CSI. 


\section{Acknowledgements}

This research was supported by the Absorption of Imported Technology and Innovation Special Funds of China (No. JJ-YJCX-04-10-2828).

\section{References}

[1] S. Haykin, "Cognitive radio: brain-empowered wireless communications", IEEE Journal on Selected Areas in Communications, vol. 23, no. 2, (2005), pp. 201-220.

[2] Q. Jin, D. Yuan, and Z. Guan, "Distributed geometric-programming based power control in cellular cognitive radio networks", Proceeding of IEEE VTC (2009).

[3] S. Huang, X. Liu and Z. Ding, "Distributed power control for cognitive user access based on primary link control feedback", Proceeding of IEEE INFOCOM, (2010), pp. 1-9.

[4] S. Sun, J. Di, and W. Ni, "Distributed power control based on convex optimization in cognitive radio networks," in Proceeding of 2nd Wireless Communications and Signal Processing (WCSP), (2010).

[5] L. Zhang, Y-C. Liang, Y. Xin, and H. V. Poor, "Robust cognitive beamforming with partial channel state information", IEEE Transactions on Wireless Communications, vol. 8, no. 8, (2009), pp. 41434153.

[6] G. Zheng G, K. Wong, and B. Ottersten, "Robust cognitive beamforming with bounded channel uncertainties”, IEEE Transactions on Signal Processing, vol. 57, no. 12, (2009), pp. 4871-881.

[7] F. Wang and W. Wang, "Robust beamforming and power control for multiuser cognitive radio network", Proceeding of IEEE GLOBECOM, (2010), pp. 1-5.

[8] T. N. Shenouda and M. Davidson, "On the design of linear transceivers for multiuser systems with channel uncertainty", IEEE Journal on Selected Areas in Communications, vol. 26, no. 6, (2008), pp. $1015-1024$.

[9] X. Gui, G. Kang and P. Zhang, "Linear precoding design in multi-user cognitive MIMO systems with cooperative feedback", IEEE Communications Letters, vol. 16, no. 10, (2012), pp. 1580-1583.

[10] S. Parsaeefard and A. R. Sharafat, "Robust distributed power control in cognitive radio networks", IEEE Transactions on Mobile Computing, vol. 12, no. 4, (2013), pp. 609-620.

[11] F. Zhao, B. Li, H.-B. Chen and X. Z. Lv, "Joint beamforming and power allocation for cognitive MIMO systems under imperfect CSI based on game theory", Wireless Personal Communications, vol. 73, no. 3, (2013), pp. 679-694,.

[12] D. Jiang, H. Zhang, and D. Yuan, "Linear precoding and power allocation in the downlink of cognitive radio networks", Proceeding of IEEE Communications, Circuits and Systems (ICCCAS), (2010), pp. 68-72.

[13] S. Boyd and L. Vandenberghe, "Convex Optimization", Cambridge, U.K.: Cambridge Univ. Press, (2004).

[14] J. Mundarath, P. Ramanathan and B. Van Veen, "A distributed downlink scheduling method for multiuser communication with zero-forcing beamforming", IEEE Transactions on Wireless Communication, vol. 7, no. 11, (2008), pp. 4508-4521.

[15] R. C. Xie, F. Richard Yu and H. Ji, "Joint power allocation and beamforming with users selection for cognitive radio networks via discrete stochastic optimization", Wireless Networks, vol. 18, (2012), pp. 481-493.

[16] L. Zhang, Y. Liang, Y. Xin and H. V. Poor, "Robust cognitive beamforming with partial channel state information", IEEE Transactions on Wireless Communications, vol. 8, no. 8, (2009), pp. 4143-4153.

[17] J. Wang, G. Scutari, and D. P. Palomar, "Robust MIMO cognitive radiovia game theory," IEEE Transactions on Signal Processing, vol. 59, no. 3, pp. 1183-1201.

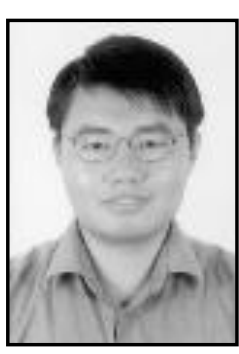

\section{Author}

Jie Chen received the Bachelor degree and Master degree in Computer_Science from Shanghai Normal University in 1997 and 2000, respectively.Now he is studying for Eng.D. Degree in Electro Mechanical Engineering in Xidian University. He received Senior Engineer in InformationTechnology in 2011. His main research inte rests include Intelligent Control, Measurement technology and His main research interests include Measurement technology, cognitive radio information security and cloud computing. 


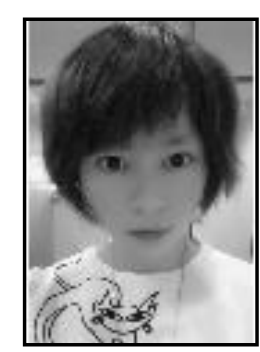

Wen Xi was born in Shanghai, China, in 1987. She received the Bachelor degree in Communication engineering from The PLA information engineering university and Master degree in Communication and information system from Guilin university of electronic science and technology, in 2010 and 2013, respectively. Her main research interests include the cognitive radio, Mobility management, information security, location management algorithm research.

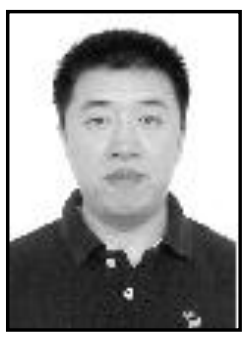

Xiaolong Sun works at is Chang jiang Computer Group Corporation , was born in 1976,received the Bachelor degrees in application of electronic from Changchun University of science and technology, in 1999. He is Hardware engineering in the company. His research interests include Electronic engineering, information security and intelligent transportation systems. 